On Electromotive Forces of Contact

This content has been downloaded from IOPscience. Please scroll down to see the full text. 1887 Proc. Phys. Soc. London 9274

(http://iopscience.iop.org/1478-7814/9/1/340)

View the table of contents for this issue, or go to the journal homepage for more

Download details:

IP Address: 130.237.165.40

This content was downloaded on 22/08/2015 at 08:32

Please note that terms and conditions apply. 
therefore, permanently twisted through twenty revolutions and then released from torsional stress. When, on heating, a certain temperature had been reached there was a sudden untwisting of the wire, followed after an interval of sereral seconds by the usual deflection of the Thomson's galvanometer, which showed that the temporary magnetic susceptibility was beginning to disappear. This experiment was repented at least six times 'with different pieces of the same kind of wire, and, invariably, with the same result-the permanent torsion, like the permanent magnetism, began to disappear suddenly at a temperature considerably below the temperature at which the temporary magnetic susceptibility began to disappear.

The contrary, however, was the case with a specimen of annealed nickel wire* of the same length as the iron but of rather less diameter. When this wire had received a few turns of permanent torsion $t$, and was afterwards heated, the loss of temporary magnetic susceptibility occurred at a much lower temperature than the rapid loss of permanent torsion; whilst Berson has shown that the permanent magnetization of nickel resembles the permanent magnetization of iron in disappearing before the temporary susceptibility.

This experiment, therefore, shows that the temperature at which permanent magnetism begins to suddenly disappear is not the temperature at which permanent torsion begins to suddenly disappear.

XL. On Electromotive Forces of Contact.

By Charles V. Burtor, B.Sc. $\ddagger$

THe principal object of this paper is to discuss the seats of the electromotive forces developed by tho contact of conductors, and by considering the transformations of energy which occur when two insulated conductors are brought into contact, an attempt is made to give a logical demonstration of some of the views put forward. In the latter part of the faper a description is given of some experiments on the

* Containing nearly 98 per cent. of nickel

+ Only two or three turns can be given before the wire breaks.

$\ddagger$ Read April $28,1888$. 
nature of the electrification of non-conductors by contact or friction.

When two conductors are brought into contact, an electromotive force $(E)$ is set up between them, which drives electricity across the junction, until the difference of their potentials* has an E.M.F. equal and opposite to E. I shall assume that $E$ is always the same so long as we keep to the same substances and the same temperature; and also that when electric equilibrium has been established each conductor is at a uniform potential throughout, except very near the junction, and that there the potential changes in general by a finite amount as we pass through a layer of very small but finite thickness, which includes the surface of separation.

Let the two conductors A and B (fig. 1) be in contact over

Fig. 1.

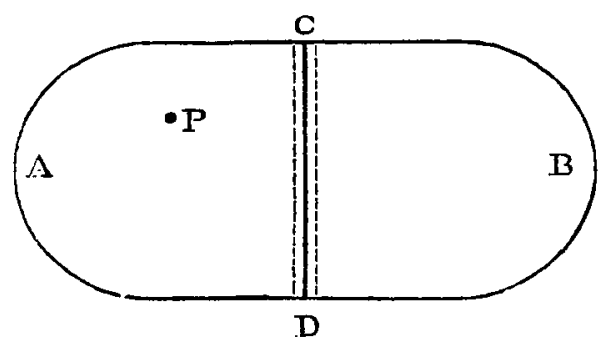

the surface CD, and let the difference of their potentials be finite. The potential is constant throughout the extent of $A$, and also throughout the extent of $B$, except within a very thin layer including the surface $C D$, which may be called the layer of variable potential, and where the potential may be supposed to change continuously from that of $A$ to that of $B$ as we pass from one side of the layer to the other. Now, consider the distribution of electricity on the system. Electric masses will be distributed on the external surfaces of $\mathbf{A}$ and $B$, but not within these surfaces, except within the layer of variable potential. Here the distribution evidently depends upon the thickness of the layer, the law of variation of

* Wherever the potential of a conductor is spoken of, the potential at any point throughout its volume is intended, and not the potential at a rery near cxterual point. I nust apologise for this unusual use of the term. 
potential across the layer, and the specific inductive capacity of $A$ and $B$. This last quantity is evidently the true or finite specific inductive capacity, and not the apparent or infinite specific inductive capacity which conductors appear to have in ordinary electrostatic experiments; for in the latter case the electric potential energy of the layer CD would be infinite.

It is usually supposed that the contact E.M.F. between two conductors is due to some molecular action between them. And my first point is to show that this action is confined to molecules within the layer of variable potential.

For consider a particle $P$ (fig. 1) within the conductor $A$, so that all around $P$ the potential is constant. Then if $P$ takes part in any way in maintaining a difference of potentials letween $A$ and $B$, it must in turn be reacted on by the electric forces which tend to equalize the potential throughout the system. Now, the only forces tending to equalize the potential are the attractions and repulsions inter se of the electric masses distributed on the conductors; and the particle $\mathrm{P}$ being situated in a region where the potential is constant, the electric force at $\mathrm{P}$ is zero.

Therefore $\mathrm{P}$ suffers no reaction from the electric masses of the system and cannot, therefore, play any part in maintaining the difference of potential.

Hence the molecular action which gives rise to a contact E.M.F. between two conductors is confined to the immediute neighliourliood of the junction.

Now, suppose that two conductors A and B (fig. 2), whose Fig. 2.

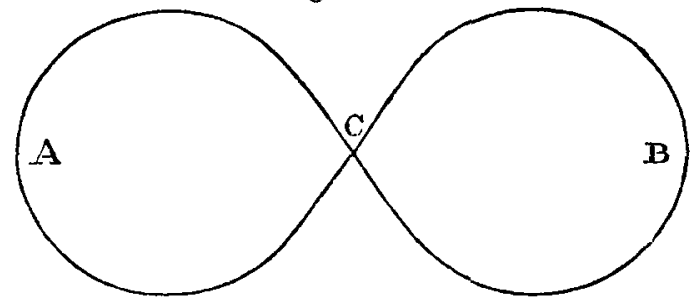

contact-difference of potentials is $P$, are originally at the same potential, and are then brought into contact at C. As soon as this is done an E.M.F. E $(=-P)$ will act between $A$ and $B$, and will drive electricity across the junction at $C$, until the difference of potentials of $\Lambda$ and $B$ becomes equal to $P$. 
At the beginning of the operation, when contact first occurs, there is no E.M.F. opposed to $E$, so that when $E$ drives a quantity $d \mathrm{M}$ of electricity across the junction, the work done is EdM. As there is no opposing E.M.F. to be overcome, the whole of this work is spent in producing heat, according to the Joule effect.

At a later stage, when the difference of potentials has become (say) $p$, the total E.M.F. between the conductors $=\mathrm{E}+p ; p$ being of course of the opposite sign to $\mathrm{E}$. Hence, when the quantity $d M$ crosses the junction, the work $(\mathrm{E}+p) d \mathrm{M}$ is spent in producing the Joule effect; while the electric potential energy of the system increases by $-p d \mathrm{M}$; so that the total work done on $d \mathrm{M}$ by the E.M.F. $\mathrm{E}=\mathrm{E} d \mathrm{M}$.

Hence, if in order to establish the difference of potentials $P$ between $A$ and $B$, the quantity $M$ of electricity must cross the junction, the work done by the E.M.F. E during the operation is E.M., half this work being spent in producing the Joule effect, and half in raising the electrostatic potential energy of the system.

But the E.M.F. $E$ is due to molecular action in the immediate neighbourhood of the junction, and, therefore, when the E.M.F. E does work by causing a displacement of electricity, a corresponding amount of molecular energy is absorbed at the junction.

Now, $\mathrm{E}$ being finite, suppose that the surface of contact at the junction $\mathrm{C}$ (fig. 2) is very small, and that the electrostatic capacities of the conductors are very great. Then $\mathbf{M}$ will be very great, and so will EM. In fact, keeping the junction as small as we please, we may increase $E M$ indefinitely by increasing the electrostatic capacity of the system.

But, by hypothesis, the E.M.F. E will always be maintained so long as we keep to the same substances, and maintain them at the same constant temperature.

Hence, when the E.M.F. E does work, the molecular energy absorbed is of such a nature that it can be supplied in indefiuite amount by a small finite junction maintained at a constant temperature.

There are only two kinds of energy which fulfil this condition:-(1) Heat; (2) Chemical action at the junction. 
First, suppose that the conductors A and B (fig. 2) are incapable of acting chemically upon one another. Then for every quantity $d \mathbf{M}$ of electricity which crosses the junction in the direction of the E.M.F. E, this E.M.F. does work EdM, and an equivalent amount of heat is absorbed at the junction. And, conversely, if electricity $d \mathbf{M}$ cross the junction in the opposite direction, work will be done against those mclecular agencies at the junction which maintain the E.M.F.E, i.e. the work done on the E.M.F. E will appear in the form of heat.

Hence, the true contact E.M.F. between two chemically inactive conductors is equal to their coefficient of the Peltier effect expressed in absolute measure.

An argument sometimes advanced against this proposition is substantially as follows (I quote from Professor Lodge's paper in the Phil. Mag. for April 1885, p. 260) :-" When Q units of electricity are transmitted against a force $E$, work $E Q$ is done; also when they are transmitted up a difference of potential $V^{1}-V$, work $Q\left(V^{1}-V\right)$ is done; but in an open circuit containing an electromotive junction, $V^{1}-V$ is produced by and is equal to $\mathrm{E}$. Hence, at an electromotive junction no work need be done by a current; in other words, the existence or non-existence of a Peltier effect has nothing to do with the existence or non-existence of a local E.M.F."

But although on an open circuit, there are two equal and opposite E.M.F.'s at the junction, and consequently there is no resistance to the flow of electricity in either direction, still the E.M.F. which acts in the direction of the flow will do work upon the opposing E.M.F.

- A mechanical example may make this clearer. If a particle be acted upon by two equal and opposite forces, each of one dyne, the smallest possible force will be able to displace the particle in any direction ; but if the particle be displaced one centimetre in the direction of one of the dyne-forces, this force will do just one erg of work upon the other force.

Now, at the junction between two chemically inactive conductors, the two E.M.F.'s are (1) an E.M.F. E due to a tendency to absorption of heat-energy at the junction, and transformation of the absorbed energy inti clectrical energy

* Professor Lodge refutes this by n hvdrostatic analogy. 
by displacement of electricity across the junction, and (2) an E.M.F. P $(\stackrel{-E}{=})$ due tu difference of potentials between the conductors.

Hence, if electricity $M$ cross the junction in the direction of the E.M.F. E, heat-cnergy EM is alsorbed in order to drive the quantity $M$ up the step of potential; while if electricity $\mathrm{M}$ cross the junction in the opposite direction, energy EM will be given out in descending the step of potential and will appear in the form of heat.

Returning now to the consideration of the conductors $\mathbf{A}$ and $B$ (fig. 2), suppose that they are capable of acting chemically upon one another. In this case the energy required to establish the difference of potentials between $A$ and B may be supplied at the junction either in the form of heat absorbed there or chemical action taking place. For the sake of simplicity let the Peltier E.M.F. be zero, so that we have only to deal with a chemical E.M.F. It will also be simplest to consider one of the conductors to be a solid and the other a liquid, so that any chemical action which may take place will not modify the nature of the subtances in contact.

For every quantity $M$ of electricity which crosses the junction in the direction of the E.M.F. chemical energy EM will be absorbed, i.e. an amount of chemical action proportional to M will take place.

This is in accordance with Faraday's Electrolytic Law.

Professor Lodge considers that the E.M.F. between chemically active substances is due to a tendency to chemical action. But mere tendency to chemical action at a finite junction could not be the source of an indefinite amount of energy, and could not, therefore, account for the phenomena in the case discussed above. There must be actual cbemical action. It appears in fact (excluding all idea of a Peltier E.M.F.) that when two chemically active conductors are brought into contact, electricity in general crosses the junction, and establishes a difference of potential between them, and the smount of chemical action which takes place is precisely the equivalent of the electricity which crosses the junction determined in accordance with Faraday's Electrolytic Law.

Let $\mathrm{K}=$ number of absolute units of energy evolved when one gram of (say) the substance $A$ enters into the kind of combination which takes place in the case concidered. 
Let $k=$ number of grams of $\mathrm{A}$ which enters into combination per unil flow of electricity.

Then for every unit of electricity which crosses the junction, $\mathrm{E}$ units of chemical energy are absorbed ; $\therefore \mathrm{E} / \mathrm{K}$ grams of $\mathrm{A}$ are dissolved.

$$
\therefore \mathrm{E} / \mathrm{K}=k \text { or } \mathrm{E}=k \mathrm{~K} \text {. }
$$

That is, the E.M.F. is erual to the energy of combination of one electrochemical equivalent.

Of course this is only the case where all the energy of the chemical action is converted into electrical energy ; as in the case of pure zinc dipped in dilute sulphuric acid. From this it follows that in the case of the typical cell, for instance, where pure zinc and pure copper are immersed in dilute sulphuric acid, the principal E.M.F is at the contact of the zinc and acid, an opposing E.M.F. being set up at the contact of acid and copper.

If we construct a cell consisting of zinc and carbon dipping in dilute $\mathrm{H}_{2} \mathrm{SO}_{4}$, there will be no power of chemical action at the liquid-carbon junction, so that the only E.M.F. there is a Peltier E.M.F. Neglecting Peltier E.M.F.'s altogether, the E.M.F. of such a cell would be equal to that of the zincliquid junction.

The apparent contact E.M.F.'s of metals, as measured in air, must be due chiefly to the air-metal contacts, since a metalmetal contact can only be the seat of a Peltier E.M.F. It also appears that if the apparent E.M.F. between two metals were measured inductively in some chemically inactive gas (or liquid), the result would be the sum of three Pelticr E.M.F.'s, and would probably be small. There even seems to be some reason for supposing it to be zero.

Fig. 3.

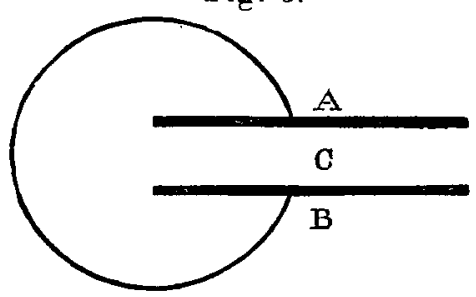

For consider a circuit consisting of two metals $\mathbf{A}$ and $B$ (fig. 3) and an inert gas C, all at the same temperature. Suppose that there is on the whole a finite E.M.F. in the 
circuit $\mathrm{ACBA}, i$. e. that the potential of the gas $\mathrm{C}$ varies as we pass from the plate $A$ to the plate $B$. Now, it is evident that if the gas $\mathrm{C}$ has any trace of conductivity, or any power whatever of equalizing potentials by convection, a feeble current will flow continuously around the circuit ACBA, and will derive its energy from absorption of heat at one or more of the junctions, which would be a violation of the Second Law of Thermodynamics. If the metal A were furnished with points as fine as possible, and if these points were brought within an extremely small distance of $\mathrm{B}$, they would greatly favour any conductive or convective tendency that might exist; and if by such an arrangement a convective discharge of only one molecule of electricity in a century were established, it would constitute a violation of the Second Law of Therinodynamics.

If we admit that in an inert gas $C$ the difference of potential between the layers in contact with $A$ and $B$ is finite, we must assume that the gas is an absolutely perfect insulator for such an E.M.F. Even if this property is assumed it does not follow that Volta's Law is inapplicable to the case of gases and metals.

I have endeavoured to show how a Peltier E M.F. depends on a tendency to absorb heat-energy and convert it into electrical energy. Now from the law of successive contacts of chemically inactive conductors, it is evident that, the temperature being fixed, we can connect with each substance a constant (called its "Peltier-constant") such that the E.M.F. between any two inactive conductors is equal to the difference of their respective constants, which is only another way of stating Volta's Law.

Now it seems not unlikely that the Peltier constant of a given conducting substance, at a given temperature, depends only on the size, form, \&c. of its molecules, and the nature of their heat-motion; and, if so, each non-conductor should also have its Peltier constant, since the conditions are essentially the same in the two cases.

Thus the law of successive contacts might hold for chemically inactive non-conductors as well as for conductors.

It might even be that the law is universally true so far as Peltier E.M.F.'s only are concerned; but of course these are only speculations.

VOL. IX. 
Tho following are somo analogous properties of Poltier W.M.F.'s and chemical E.M.F.'s arranged respectively in parallel columns :-

Pfiltier E.Mi.F.

1a. When the two substances originally at the siane potential are brought into contact an E.M.F. $E_{1}$ is established across the junction; this E.M.F. continues to displace electricity across the junction until the E.M.F. due to difference of potentials $=-\mathrm{E}_{2}$

$2 a$. The energy required to establish the difference of potentials is supplied by heat absorbed at the iunction.

$3 a$. For every quantity $\mathrm{M}$ of electricity which crosses the junction in the direction of the E.M.F. $\mathrm{E}_{1}$, a quantity of heat-energy $\mathbf{E}_{\mathbf{r}} \mathbf{M}$ is absorbed at the junction.

4a. For every quantity MI of electricity which crosses the junction against the E.M.F. $E_{1}$, heat-energy $\mathbf{E}_{1} \mathbf{M}$ is given out at the janction.

5a. The heat absorbed at the junction during the operation (la) is the equivalent of the electricity which crosses the junction, determined in accordance with the laws of the Peltier effect.
Chrmical E.M.F.

1b. When the two substances originally at the same potential are brought into contact an L.M.F. $\mathbf{E}_{2}$ is established across the junc. tion; this E.M.F. continues to displace electricity across the junction until the E.ML.F. due to difference of potentials $=-E_{2}$.

$2 b$. The energy required to establish the difference of potentials is sup. plied by chemical action talzing place at the junction.

32. For every quantity $\mathrm{MI}$ of elec. tricity which crosses the junction in the direction of the E.M.F. $\mathrm{E}_{2}$, a quantity of chemical energy $\mathbf{E}_{z} \mathbf{M}$ is absorbed at the junction.

4b. For every quantity M of electricity which crosses the junction against the E.M.F. $E_{2}$ energy $\mathbf{E}_{2} \mathbf{M}$ is given out at the junction, and appears either as chemical-energy (electrolysis) or as heat.

5b. The chemical action at the juuction during the operation (1b) is the equivalent of the electricity which crosses the junction, determined in accordance with Faraday's Electrolytic Law.

Sir W. Thomson has deduced a lower limit to the size of atoms by a method which is well known. Professor Lodge points out that the limit cannot be assigned with certainty, until the true contact-difference of potentials of the substances concerned is known. But there is another point to be considered. The method assumes that there is a kind of affinity between the molecules of the two metals concerned; that on contact being made, this affinity gives rise to an E.M.F. which establishes a difference of potential, the necessary energy being furnished by the partial satisfaction of the said 
affinity. The method further assumes that when the metals are fused together it is this same affinity which, being satisfied as completely as possible, gives rise to an evolution of heat. But if (as appears from what precedes) the energy required to produce the difference of pctentials is supplied by absorption of heat at the junction, and if the contact E.M.F. is due to a tendency to convert heat-energy into electrical energy, then the hent evolved on fusing the metals together can have no direct connexion with E.M.F.'s of contact, and the method would not apply to the case of two metals.

It would, however, obviously apply where the E.M.F. was due to chemical affinity, as in the case of zinc and oxygen proposed by Professor Lodge (Phil. Mag. May 1885, p. 363). In all these investigations the electrostatic capacities are calculated on the assumption that the specific inductive capacity of the conductors is unity. I have not been able to find any experimental justitication for this assumption.

\section{Electrification of Non-conductors.}

When two non-conductors are brought into contact, there are other sources of energy which may cause electrification. For if the bringing of two free surfaces into contact, or the striking or rubbing of the bodies together, should give rise to electrification, the charges so produced would remain separated, owing to the insulating properties of the substances. This source of electrification is absent in conductors, as any electrification produced between them by sudden violenco would be destroyed by conduction, the final difference of potentials between the bodies being always that due to a definite contact E.M.F.

The difference between the two cases may be illustrated by considering the case of a spring. If a force be applied to the spring a definite compression will ensue, supposing that there is no friction; and if by any temporary impulse the compression be increased or diminished, it will regain its former value when the impulse ceases to act. The inverse of the strength of the spring represents the capacity of the system, the force compressing the spring the E.M.F. of contact, the distance through which the spring is compressed the quantity of electricity displaced, and the potential energy of 
the compressed spring the electrostatic potential energy of the two conductors.

If we suppose compression or extension of the spring to be accompanied by friction, a compression effected by a sudden shock will be maintained. This illustrates what perhaps takes place with non-conductors. In order to test which view is the more correct, $I$ have made a few experiments on the electrification of glass and ebonite when dipped into mercury. This method of experimenting seemed to offer several advantages. The mercury being permanently connected to earth was always at a known potential (zero), and it was only necessary to measure the charge of the ebonite or glass. Then, again, contact was obtained over the whole surface submerged, with very little mechanical violence when desired ; and the conditions of contact could be considerably varied by using more or less suddenness and violence and longer or shorter duration of contact.

Fig. 4.

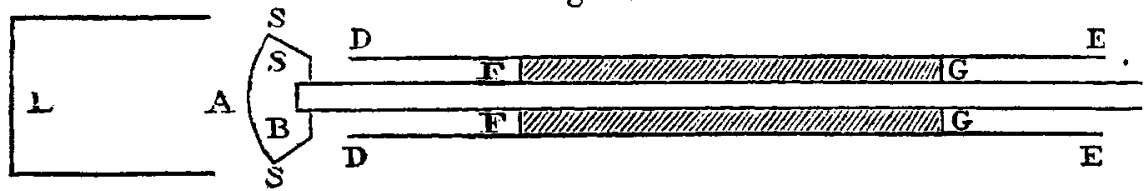

The ebonite or glass employed consisted of a small circular slab S (fig. 4) with a convex surface A, which was attached to one end of a rod $\mathrm{BC}$ of the same material. This rod was encased in a brass tube DE, inside which it was fastened by means of packing FG. The length $\mathrm{BF}$ of ebonite or glass served to insulate the slab $A$. In the case of the glass the length $\mathrm{BF}$ was varnished. The charge on $\mathrm{A}$ was measured by means of the conductor $\mathrm{L}$ connected to a Thomson electrometer. A was placed near to $\mathrm{L}$, which was put to earth and then insulated. On withdrawing A, a deflection was produced proportional to the charge on $A$. The movement of $\mathrm{A}$ was controlled by making the tube $\mathrm{DE}$ slide in metal bearings between fixed stops. The tube $\mathrm{DE}$ being of metal, no electrification was produced by friction in the bearings. The following results were obtained :- 


\section{(a) Ebonite and Mercury.}

DEFLECTION.

Dipped once and quickly withdrawn. . . +10 to +12 very quickly withdrawn . . +15

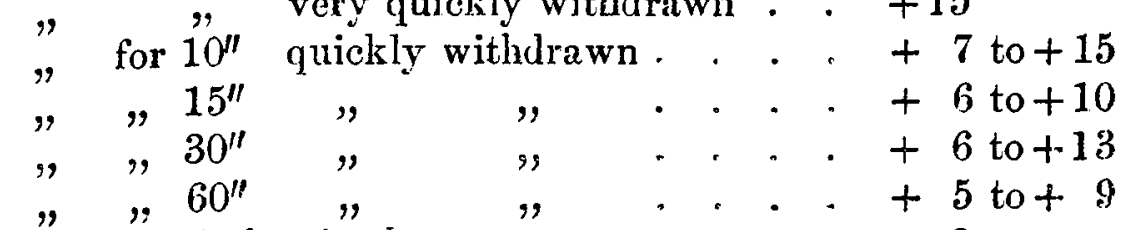

30 rapid splashes in the mercury . . . . - 6

50 not very rapid splashes in the mercury - + 7

100 rapid

200 "

$" \quad " \quad " \quad$ " $\quad$ " $\quad-11$

(b) Glass and Mercury.

DEFLECTION.

Dipped once quietly . . . . . . . + +6 to +10

20 rapid splashes . . . . . . . . . -7

$\begin{array}{rll}50 & & \\ 60 & " & \\ 90 & " & \\ 100 & " & \end{array}$

The above results, it will be seen, are very irregular, owing no doubt to the fact that all the circumstances of contact could not be accurately known. These irregularities, and especially the variable sign of the electrification, make it clear that the effects are not due to a true and definite contact E.M.F., but are dependent in some degree on the manner in which the surfaces are brought together and separated. The experiment of Canton in 1753 (where the rough and polished parts of a glass tube becamé oppositely electrified when rubbed with the same cloth) seems to point in the same direction.

Professors Ayrton, Schuster, S. P. Thompson, and J. Perry discussed the points raised, and it was considered that direct experiment on contact electromotive force in a very perfect vacuum could alone decide the question.

This experiment seems almost as impracticable as a direct measurement of the size of a molecule. Yet the two questions may be equally capable of solution by inductive reasoning. The question is whether the reasoning given above is or is not conclusive.-C. V. B. 